\title{
Intra-Abdominal Nocardiosis-Case Report and Review of the Literature
}

\author{
Lucas Tramèr ${ }^{1}$, Kirsten D. Mertz ${ }^{2}$, Rolf Huegli ${ }^{3}$, Vladimira Hinic ${ }^{4}$, Lorenz Jost ${ }^{1,5}$, \\ Felix Burkhalter ${ }^{1,6}{ }^{\mathbb{D}}$, Sebastian Wirz ${ }^{1,7}$ and Philip E. Tarr $1,7, *$ (D) \\ 1 University Department of Medicine, Kantonsspital Baselland, University of Basel, 4101 Bruderholz, \\ Switzerland; lucas.tramer@ksbl.ch (L.T.); lorenz.jost@ksbl.ch (L.J.); felix.burkhalter@ksbl.ch (F.B.); \\ sebastian.wirz@ksbl.ch (S.W.) \\ 2 Cantonal Institute of Pathology, 4410 Liestal, Switzerland; kirsten.mertz@ksbl.ch \\ 3 Department of Radiology and Nuclear Medicine, Kantonsspital Baselland, 4101 Bruderholz, Switzerland; \\ rolf.huegli@ksbl.ch \\ 4 Clinical Bacteriology and Mycology, Laboratory Medicine, University Hospital Basel, 4031 Basel, \\ Switzerland; vladimira.hinic@usb.ch \\ 5 Oncology Service, Kantonsspital Baselland, 4101 Bruderholz, Switzerland \\ 6 Nephrology Service, Kantonsspital Baselland, 4101 Bruderholz, Switzerland \\ 7 Infectious Diseases Service, Kantonsspital Baselland, 4101 Bruderholz, Switzerland \\ * Correspondence: philip.tarr@unibas.ch; Tel.: +41-61-436-2212; Fax: +41-61-436-3670
}

Received: 11 May 2020; Accepted: 2 July 2020; Published: 7 July 2020

check for updates

\begin{abstract}
Nocardiosis is primarily an opportunistic infection affecting immunosuppressed individuals, in whom it most commonly presents as pulmonary infection and sometimes cerebral abscesses. Isolated abdominal or retroperitoneal nocardiosis is rare. Here, we report the second case, to our knowledge, of isolated abdominal nocardiosis due to Nocardia paucivorans and provide a comprehensive review of intra-abdominal nocardiosis. The acquisition of abdominal nocardiosis is believed to occur via hematogenous spreading after pulmonary or percutaneous inoculation or possibly via direct abdominal inoculation. Cases of Nocardia peritonitis have been reported in patients on peritoneal dialysis. Accurate diagnosis of abdominal nocardiosis requires histological and/or microbiological examination of appropriate, radiologically or surgically obtained biopsy specimens. Malignancy may initially be suspected when the patient presents with an abdominal mass. Successful therapy usually includes either percutaneous or surgical abscess drainage plus prolonged combination antimicrobial therapy.
\end{abstract}

Keywords: nocardiosis; Nocardia paucivorans; infection; abdominal; retroperitoneal; abscess; immunosuppression; malignancy

\section{Introduction}

Nocardia are gram-positive, aerobic, filamentous bacteria that most often cause pulmonary infection in immunosuppressed hosts, while isolated abdominal or retroperitoneal nocardiosis is rare [1]. In extrapulmonary nocardiosis, malignancy is often suspected due to the presence of abdominal or cerebral masses [2-6], which can lead to misdiagnosis. Additionally, accurate diagnosis may be challenging due to slow growth of nocardia species in culture $[7,8]$.

Here, we report the second case, to our knowledge, of isolated abdominal nocardiosis due to Nocardia paucivorans. We also provide a comprehensive review of the literature, summarizing the information on 39 previously published cases of intra-abdominal and retroperitoneal nocardiosis and 12 cases of peritoneal dialysis-related nocardia peritonitis [2,5,6,8-53]. Finally, we will review published cases of infection with Nocardia paucivorans, a rare nocardial species. 


\section{Illustrative Case Report}

An 81-year-old man with diffuse large B-cell lymphoma presented in January 2019 with abdominal pain and night sweats. In 2009, he initially presented with abdominal pain, weight loss, night sweats, and fever. Abdominal CT showed a mesenteric mass adjacent to the ileocecal area. Histopathological examination of a surgically obtained biopsy specimen showed the presence of CD20- and CD3-positive lymphoma cells, a perifocal lymphohistocytiary inflammatory infiltrate as well as necrotic lymphoma tissue. A bone marrow biopsy was free of lymphoma cells. Weekly rituximab was administered for 4 weeks, in combination with vincristin and bleomycin for the first 3 weeks. Rituximab plus cyclophosphamide, hydroxydaunorubicin, oncovin and prednisolone (R-CHOP) was administered for a total of 6 cycles until full remission was achieved in February 2010. After a successfully treated low-grade nodal relapse in 2017, the patient remained asymptomatic until February 2018 when persistent hypogammaglobulinemia was first noted and intravenous immune globulin was given at 4-week intervals. Since the last relapse in 2017, the patient had received no immunosuppressive therapy.

In January 2019, the patient presented with generalized weakness, night sweats, and intermittent abdominal pain for the past 3 weeks, as well as fever and diarrhea for 1 week. On physical examination, the abdomen was soft and without tenderness. CRP $(197 \mathrm{mg} / \mathrm{L})$ and the white blood cell count $\left(18,100 / \mathrm{mm}^{3}\right)$ were elevated. A chest X-ray showed no signs of pulmonary infection, and on CT scan of the head there were no signs of cerebral nocardiosis. Abdominal CT showed a heterogeneous mass in the right adrenal region (Figure 1), with FDG-PET-CT showing locally increased FDG uptake and central necrosis. A biopsy specimen, radiologically obtained with CT-guidance revealed numerous granulocytes and histiocytes but no signs of malignancy, suggesting a retroperitoneal abscess.

A pigtail catheter was placed under CT-guidance for drainage of the abscess (Figure 1) and empiric antibiotic therapy with intravenous amoxicillin/clavulanic acid was initiated. Gram stain of the drainage fluid showed filamentous bacteria, consistent with Nocardia species (Figure 2). Culture grew Nocardia paucivorans, susceptible in vitro to ceftriaxone, imipenem, amikacin, trimethoprim-sulfamethoxazole (TMP-SMX), ciprofloxacin and minocycline and resistant to amoxicillin/clavulanic acid. Nocardia paucivorans was identified by matrix-assisted laser desorption ionization time-of-flight mass spectrometry (MALDI-TOF MS). The mass spectra were acquired using the Microflex LT system and analyzed with the MALDI Biotyper Compass 4.1 software (Bruker Daltonics, Bremen, Germany). The identification was confirmed by partial 16S rRNA gene sequencing as described previously [54]. Antimicrobial susceptibility testing was performed with Liofilchem ${ }^{\circledR}$ MIC Test Strips (Liofilchem, Roseto degli Abruzzi, Italy) on Mueller Hinton agar $+5 \%$ horse blood $+20 \mathrm{mg} / \mathrm{l} \mathrm{B}-\mathrm{NAD}$ (MHF; bioMérieux, Marcy-l'Étoile, France), and the minimal inhibitory concentrations (MICs) were interpreted according to CLSI guidelines [55]. PCR and culture were negative for mycobacteria. The route of acquisition of Nocardia paucivorans in this patient could not be identified.

Antibiotic therapy was changed to intravenous trimethoprim-sulfamethoxazole and amikacin, and the patient clinically improved. The trough-level of amikacin was monitored and kept in the recommended range. However, the patient developed acute renal failure (eGFR decrease from 50 to $14 \mathrm{~mL} / \mathrm{min}$ ) and metabolic acidosis. This prompted the change of antimicrobial treatment to ceftriaxone and minocycline, and renal function recovered.

Due to considerable residual abscess despite external drainage, surgical abscess drainage was performed and the patient further improved. He was discharged on oral minocycline and ciprofloxacin. On follow-up, six weeks postoperatively, he reported minimal abdominal pain, tolerated antibiotic therapy well, and blood inflammatory markers were normal. Antibiotic therapy was continued for a total of 6 months. On most recent follow-up, 6 months after antibiotics were discontinued, the patient was well, and abdominal ultrasound was unremarkable. 

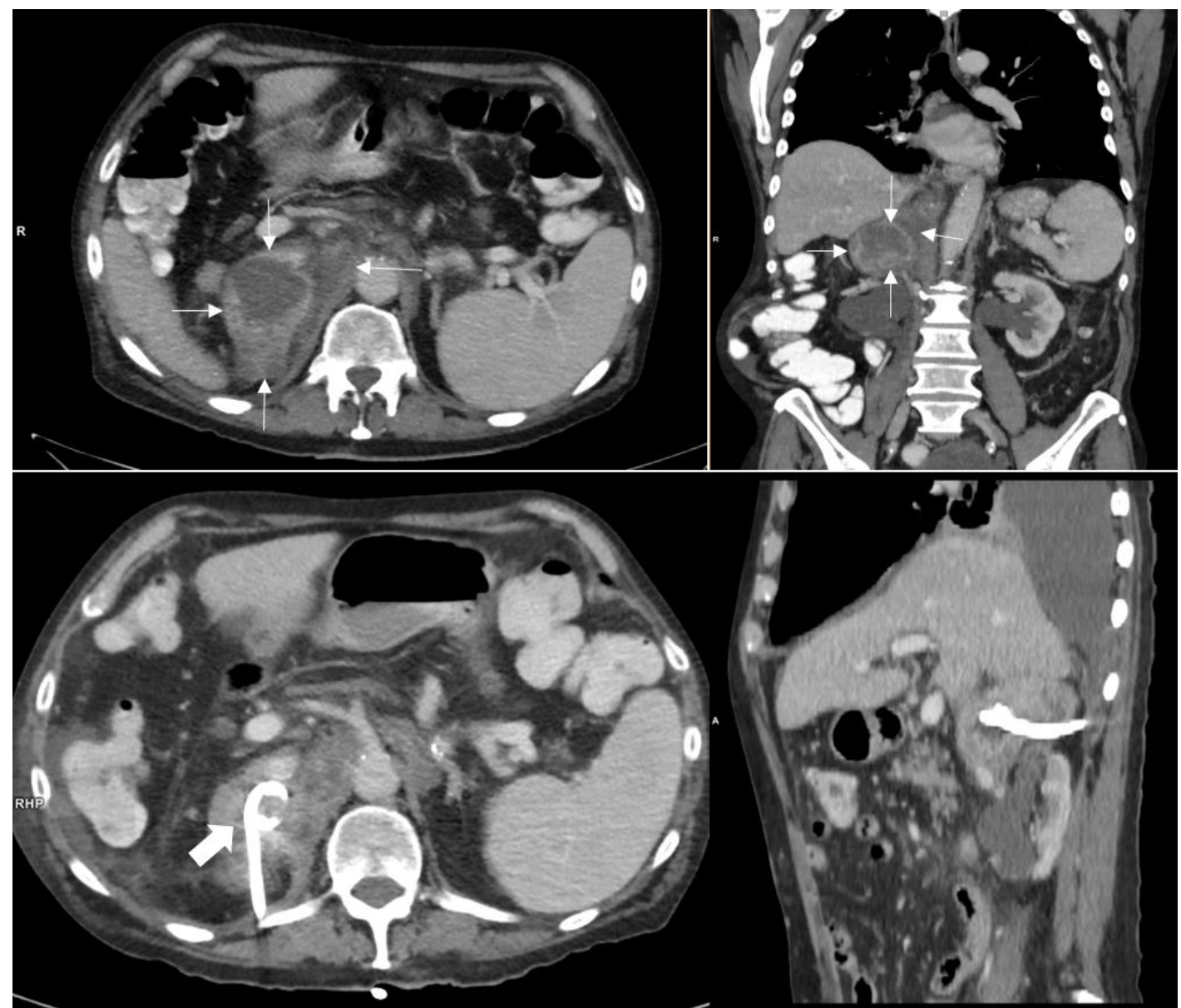

Figure 1. Upper row: $11 \times 6 \mathrm{~cm}$ retroperitoneal mass in the right adrenal loge (thin white arrows) with contact to diaphragm, liver and retroperitoneal vessels. The pararenal space is not involved. The mass shows an inhomogeneous enhancement. The right adrenal gland itself cannot be distinguished. Lower row: Mass after puncture and CT guided drainage with a pigtail catheter (thick white arrow).

\section{Methods}

We reviewed the existing literature on abdominal nocardiosis and infections due to Nocardia paucivorans. To identify patients in the published literature, several PubMed searches were performed. Search items included "nocardiosis", "nocardia", "paucivorans", "abdominal", "retroperitoneal", "CAPD", "peritonitis" and "infection". Additional cases were identified through bibliographic review of retrieved publications. Abdominal nocardiosis was defined as clinical or radiological signs compatible with nocardiosis plus isolation of Nocardia species in at least one abdominal or retroperitoneal specimen regardless of the infection's origin and extent of dissemination. Nocardia peritonitis was defined as clinical signs of peritonitis plus isolation of Nocardia species in ascites or peritoneal dialysate. $N$. paucivorans infection was defined as clinical or radiological signs compatible with nocardiosis plus isolation of $N$. paucivorans in an appropriate specimen, with clinical or radiological signs of infection. We limited our review to English and German language publications since 1966. The initial searches led to 93 publications being retrieved, of which 29 were excluded ( 26 articles published prior to 1966, 2 publications in Japanese, 1 publication in Spanish). Therefore, the present review includes 64 publications, including 49 cases of $N$. paucivorans infection, 39 cases of abdominal or retroperitoneal nocardiosis, 12 cases of nocardial peritonitis, plus our own patient with abdominal N. paucivorans infection. 


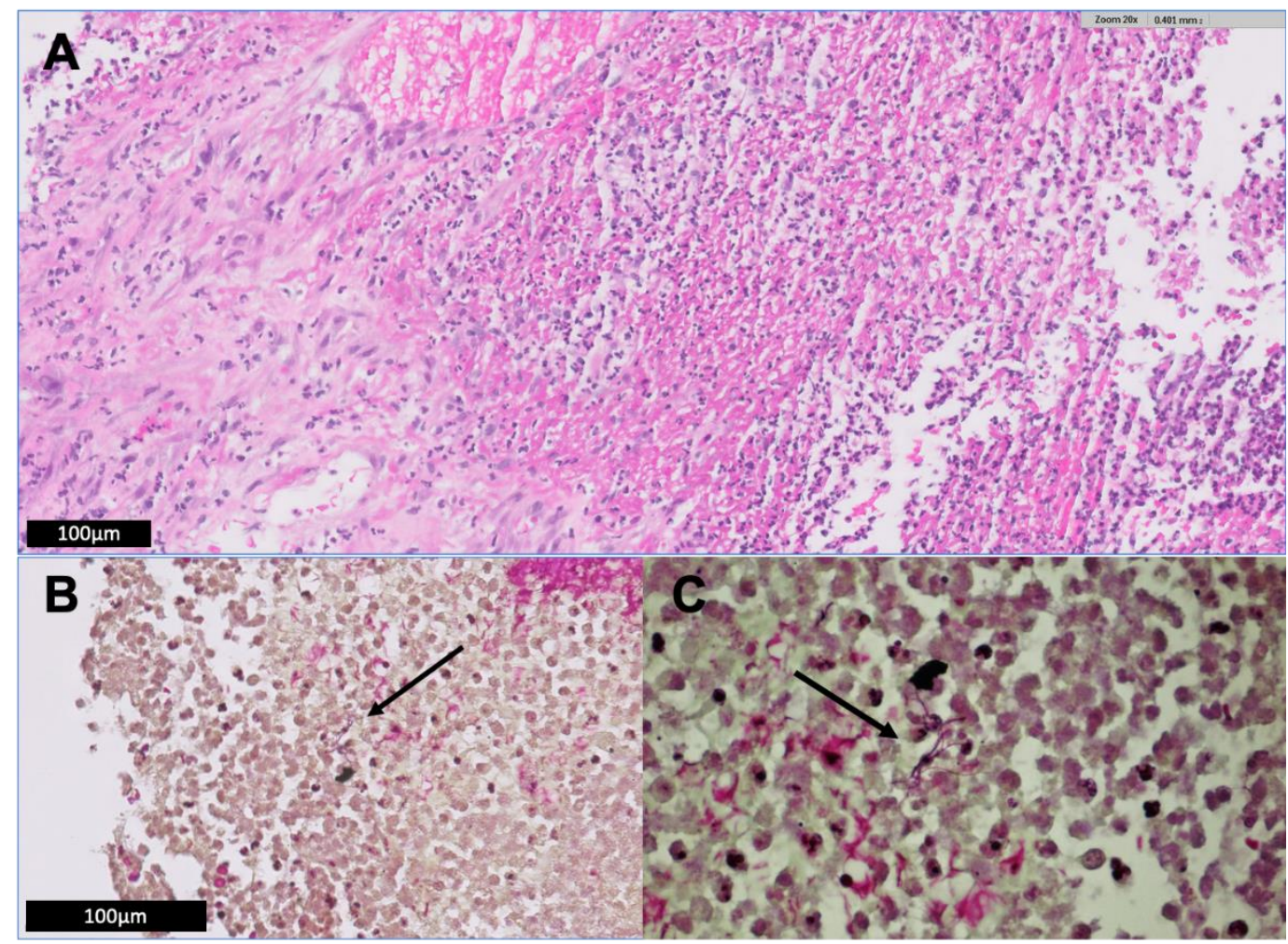

Figure 2. (A) Histologic examination of a retroperitoneal biopsy specimen demonstrates a diffuse neutrophilic infiltrate with necrosis and cellular debris. High magnification of a typical area of one of these abscesses with neutrophils, debris and liquefactive necrosis. Organisms are not visible on routine H\&E stain. (B,C) Gram stain shows filamentous bacteria (arrows), consistent with Nocardia species. The organisms are Gram-variable or weakly Gram positive, thin and filamentous, and focally concentrated.

\section{Literature Review and Discussion}

In this review of the literature, we will first discuss the presumed route of acquisition of nocardiosis based on previous case reports and reviews. Second, we will discuss previously reported infections with N. paucivorans. Third, we will concentrate on the characteristics of abdominal nocardiosis and Nocardia peritonitis.

\subsection{Route of Nocardia acquisition}

Nocardia are ubiquitously present in soil, water and decaying vegetation [56]. Infection is believed to occur mostly through inhalation or percutaneously (i.e., via local trauma, skin cuts or scrapes); intestinal inoculation has at least been suggested to occur [7]. In immunocompetent individuals (1/3 of cases), nocardiosis tends to remain localized to the site of inoculation [7]. With immunosuppression, disseminated nocardiosis may occur, clinically most often presenting as brain abscesses $[7,56,57]$. Presumably, respiratory acquisition of Nocardia in immunosuppressed patients is followed by hematologic dissemination, eventually allowing infection at distant sites [22,38]. Additionally, direct peritoneal inoculation has been reported in 12 cases of Nocardia peritonitis in patients on peritoneal dialysis [8,43-53].

\subsection{Infection Due to Nocardia Paucivorans}

N. paucivorans was first isolated in sputum in 2000 [58]. To our knowledge, 49 cases of N. paucivorans infection have been published [2-4,56,58-68], and we summarize these in Table 1. The majority (33 cases) were reported by Gray et al. from Queensland, Australia, and were 
identified retrospectively via 16S ribosomal DNA sequencing of archived biopsy specimens [63]. Unfortunately, the paper by Gray et al. lacks details regarding therapy and outcome of most patients, limiting the information to be gained from these cases. Additionally, none of the 49 previously reported cases included detailed information on the presumed route of acquisition of $\mathrm{N}$. paucivorans infection [2-4,56,58-68].

In 18 of 49 cases (37\%), no information was provided regarding the patients' immune status. Of the remaining 31 cases, 11 patients (35\%) were reported to be immunocompetent while 20 patients $(65 \%)$ were overtly immunocompromised or had an underlying clinical condition. Thus, nocardiosis with $\mathrm{N}$. paucivorans involves immunocompetent and immunocompromised patients, similar to infection with other Nocardia species $[1,7]$.

Microbiologically documented lung involvement was reported in 29 of 49 cases (59\%) of human N. paucivorans infection, while 19 cases (39\%) involved the central nervous system (mainly brain abscesses). Additional cases include skin and muscle abscesses, bacteremia and endocarditis $[3,4,56,58-$ $63,65,66,68]$. There is one case of abdominal/retroperitoneal N. paucivorans infection in the literature, a 63-year old lung transplant recipient with a renal abscess published by Roy in 2018 [2]. Our patient reported in Section 2 thus represents the second published case of intra-abdominal/retroperitoneal infection with N. paucivorans. N. paucivorans is typically susceptible in vitro to most commonly employed anti-nocardial agents [56] with TMP-SMX being used most frequently. 
Table 1. Previously published cases of Nocardia paucivorans infection.

\begin{tabular}{|c|c|c|c|c|c|c|c|c|}
\hline $\begin{array}{c}\text { Case } \\
n^{\circ} / \text { Reference }\end{array}$ & Year ** & Age & Sex & Site & Immune Status & Therapy Regimen & $\begin{array}{l}\text { Therapy } \\
\text { Duration }\end{array}$ & Outcome \\
\hline $1[58]$ & 2000 & 51 & $\mathrm{M}$ & Sputum & Chronic lung disease & NR & NR & NR \\
\hline $2[59]$ & 2002 & 40 & $\mathrm{~F}$ & Brain abscess & NR & NR & NR & NR \\
\hline $3[60]$ & 2002 & 63 & M & Brain abscess & $\begin{array}{l}\text { Low CD4+ count of } \\
\text { unknown origin }\end{array}$ & $\begin{array}{l}\text { Cef, Amp, Amk, Mer, Levo, } \\
\text { Mino }\end{array}$ & $>6$ months & survived \\
\hline $4[61]$ & 2006 & 54 & M & Sputum, BAL, lung biopsy & Immunocompetent & TMP/SMX, A/clav, Cip & 6 months & NR \\
\hline $5[62]$ & 2006 & 63 & M & Brain abscess & Immunocompetent & $\begin{array}{l}\text { Surgical resection, } \\
\text { TMP/SMX }\end{array}$ & 3 months & survived \\
\hline $6[63]$ & 2007 & 52 & $\mathrm{M}$ & Lung abscess & NR & NR & NR & died \\
\hline $7[63]$ & 2007 & 55 & M & Brain abscess & NR & NR & NR & NR \\
\hline $8[63]$ & 2007 & NR & M & Pleural fluid & NR & NR & NR & NR \\
\hline $9[63]$ & 2007 & 44 & $\mathrm{M}$ & Mediastinal lymph node & NR & NR & NR & NR \\
\hline $10[63]$ & 2007 & 78 & $\mathrm{~F}$ & Lung abscess & Chronic lung disease & NR & NR & NR \\
\hline $11[63]$ & 2007 & 50 & F & Sputum & NR & NR & NR & NR \\
\hline 12 [63] & 2007 & 41 & M & Brain abscess & Hodgkin's lymphoma & NR & NR & NR \\
\hline 13 [63] & 2007 & 53 & M & Lung abscess & NR & NR & NR & NR \\
\hline $14[63]$ & 2007 & 58 & $\mathrm{~F}$ & Brain abscess & Corticosteroid therapy & NR & NR & NR \\
\hline $15[63]$ & 2007 & 66 & $\mathrm{~F}$ & Sputum & NR & NR & NR & NR \\
\hline $16[63]$ & 2007 & 54 & M & $\begin{array}{l}\text { Endocarditis, brain abscess, } \\
\text { skin abscess }\end{array}$ & NR & NR & NR & NR \\
\hline 17 [63] & 2007 & 65 & $\mathrm{~F}$ & Sputum & NR & NR & NR & NR \\
\hline 18 [63] & 2007 & 72 & F & Lung abscess & Corticosteroid therapy & NR & NR & NR \\
\hline 19 [63] & 2007 & 66 & $\mathrm{M}$ & Brain abscess & Immunosuppression * & NR & NR & NR \\
\hline 20 [63] & 2007 & 74 & $\mathrm{M}$ & BAL fluid & NR & NR & NR & NR \\
\hline $21[63]$ & 2007 & NR & $\mathrm{M}$ & Sputum & NR & NR & NR & NR \\
\hline $22[63]$ & 2007 & 74 & $\mathrm{M}$ & Skin abscess & NR & NR & NR & NR \\
\hline $23[63]$ & 2007 & 57 & $\mathrm{M}$ & Brain abscess, pneumonia & NR & NR & NR & NR \\
\hline $24[63]$ & 2007 & 80 & $\mathrm{M}$ & Lung, skin & NR & NR & NR & NR \\
\hline $25[63]$ & 2007 & 62 & $\mathrm{M}$ & Skin & Immunocompetent & NR & NR & NR \\
\hline $26[63]$ & 2007 & 87 & $\mathrm{M}$ & Sputum & NR & NR & NR & NR \\
\hline $27[63]$ & 2007 & 77 & $\mathrm{~F}$ & BAL fluid & Immunocompetent & NR & NR & NR \\
\hline 28 [63] & 2007 & 50 & $\mathrm{M}$ & Sputum & Lung cancer & NR & NR & NR \\
\hline $29[63]$ & 2007 & 67 & $\mathrm{M}$ & Brain abscess & Diabetes mellitus & NR & NR & NR \\
\hline $30[63]$ & 2007 & 67 & $\mathrm{M}$ & Sputum & Immunocompetent & NR & NR & NR \\
\hline
\end{tabular}


Table 1. Cont.

\begin{tabular}{|c|c|c|c|c|c|c|c|c|}
\hline $\begin{array}{c}\text { Case } \\
\mathbf{n}^{\circ} / \text { Reference }\end{array}$ & Year ** & Age & Sex & Site & Immune Status & Therapy Regimen & $\begin{array}{l}\text { Therapy } \\
\text { Duration }\end{array}$ & Outcome \\
\hline $31[63]$ & 2007 & 47 & $\mathrm{~F}$ & Sputum & NR & NR & NR & NR \\
\hline 32 [63] & 2007 & 66 & $\mathrm{~F}$ & Sputum & Previous lung infection & NR & NR & NR \\
\hline 33 [63] & 2007 & 46 & M & Sputum & Immunocompetent & NR & NR & NR \\
\hline $34[63]$ & 2007 & 79 & M & Pleural fluid & Corticosteroid therapy & NR & NR & NR \\
\hline $35[63]$ & 2007 & 76 & M & Pleural fluid & Immunocompetent & NR & NR & NR \\
\hline 36 [63] & 2007 & 61 & M & Skin abscess & NR & NR & NR & NR \\
\hline $37[63]$ & 2007 & 60 & M & Blood & Chemotherapy & NR & NR & NR \\
\hline 38 [63] & 2007 & 53 & M & BAL fluid and skin lesion & $\begin{array}{l}\text { Smoking and other drug } \\
\text { abuse, Hepatitis } C\end{array}$ & Tica/clav, Rox, TMP/SMX & 12 months & survived \\
\hline $39[56]$ & 2014 & 50 & M & $\begin{array}{l}\text { M. iliopsoas, brain, lung, } \\
\text { mediastinal lymph nodes }\end{array}$ & $\begin{array}{l}\text { Smoking and other drug } \\
\text { abuse }\end{array}$ & TMP/SMX, Imi, Mox & 12 months & survived \\
\hline $40[64]$ & 2015 & 70 & M & Brain abscesses & $\begin{array}{l}\text { Multiple Myeloma under } \\
\text { chemotherapy }\end{array}$ & TMP/SMX, Mer, Cip & 12 months & survived \\
\hline $41[65]$ & 2016 & 50 & M & Brain abscesses & $\begin{array}{l}\text { Smoking and other drug } \\
\text { abuse }\end{array}$ & TMP/SMX, Imi, Line, Mox & 12 months & survived \\
\hline $42[66]$ & 2016 & 80 & $\mathrm{~F}$ & Brain and lung abscess & Immunocompetent & $\begin{array}{l}\text { Surgical resection, } \\
\text { TMP/SMX, Cef }\end{array}$ & 9 months & survived \\
\hline $43[66]$ & 2016 & 50 & M & Spinal cord, lung & Alcohol abuse & $\begin{array}{l}\text { Surgical resection, } \\
\text { TMP/SMX, Mer, Cef }\end{array}$ & 9 months & survived \\
\hline $44[66]$ & 2016 & 59 & M & Brain and lung abscess & Immunocompetent & TMP/SMX, Mer & 12 months & survived \\
\hline $45[67]$ & 2016 & 54 & M & Brain abscess & Immunocompetent & $\begin{array}{l}\text { Cef, Met, Mer, Line, Imi, } \\
\text { Van, Rif, TMP/SMX }\end{array}$ & 21 weeks & survived \\
\hline $46[3]$ & 2018 & 61 & M & Brain abscess & $\begin{array}{l}\text { Myasthenia gravis, } \\
\text { corticosteroid therapy }\end{array}$ & $\begin{array}{l}\text { TMP/SMX, Cef, Met, Line, } \\
\text { Levo }\end{array}$ & 11 months & survived \\
\hline $47[2]$ & 2018 & 66 & $\mathrm{~F}$ & Renal abscess & $\begin{array}{l}\text { Post Lung } \\
\text { Transplantation }\end{array}$ & TMP/SMX, Imi & 9-12 months & survived \\
\hline $48[68]$ & 2018 & 42 & M & Lung empyema & Suspected silicosis & TMP/SMX & NR & survived \\
\hline $49[4]$ & 2019 & 52 & $\mathrm{~F}$ & Brain abscess & Immunocompetent & $\begin{array}{l}\text { Surgical resection, Cef, } \\
\text { TMP/SMX, Levo }\end{array}$ & 13 months & survived \\
\hline $\begin{array}{l}50 \text { [present } \\
\text { report] }\end{array}$ & & 81 & M & Abscess in right adrenal loge & $\begin{array}{l}\text { B-Cell Lymphoma, } \\
\text { Hypogammaglobulinemia }\end{array}$ & $\begin{array}{l}\text { Surgical resection, A/clav, } \\
\text { TMP/SMX, Amk, Cef, Mino, } \\
\text { Cip }\end{array}$ & 6 months & survived \\
\hline
\end{tabular}

Abbreviations: Amk = amikacin; Amp = ampicillin; A/clav = amoxicillin + clavulanic acid; BAL = bronchoalveolar lavage; Cef = ceftriaxone; Cip = ciprofloxacin; Imi = imipenem; Levo = levofloxacin; Line = linezolid; Mer = meropenem; Met = metronidazol; Mino = minocycline; Mox = moxifloxacin; NR = not reported; Rif = rifampicin; Rox = roxifloxacin; Tica/clav = ticarcillin + clavulanic acid; TMP/SMX = trimethoprim-sulfamethoxazole; Van = vancomycin; ${ }^{*}$ not further specified; ** Year of publication. 


\subsection{Abdominal/Retroperitoneal Infection Due to Nocardia Species}

There are 39 cases of abdominal/retroperitoneal nocardiosis reported in the English and German language literature since 1966 [2,5,6,9-42] (Tables 2 and 3). In 16 of 39 cases (41\%), concurrent involvement of the lungs and/or brain was recorded (Table 3). Isolated abdominal nocardiosis with no lung or brain involvement was reported in 23 previous cases (59\%) (Table 2).

$N$. asteroides and N. farcinica (10 cases each) represent the most commonly isolated species. The route of Nocardia acquisition leading to abdominal or retroperitoneal infection remains unclear in most case reports. Some authors of published abdominal nocardiosis cases have speculated on the route of acquisition, which may have included inhalation of infected dust followed by hematogenous spread of Nocardia species (Table 2, case 23) [26], and acquisition via infected pet dogs with skin lesions due to distemper virus (Table 2, case 22) [25]. While direct intestinal inoculation has been suggested to occur [7], involvement of the intestines (jejenum, ileum or colon) was observed in only 6 cases $(15 \%)$, suggesting that hematogenous spread from an extraabdominal inoculation site seems the more likely scenario in most cases.

Patients with impaired cell-mediated immunity such as solid organ transplant recipients and patients with advanced HIV infection may be at particular risk for contracting disseminated nocardiosis [7,57]. Risk factors for abdominal nocardiosis remain a matter of speculation. One patient (Table 2, case 14) had colon cancer with a resulting bowel perforation and abscess at this site [42]. $\mathrm{N}$. veterana was cultured from the abscess, and the authors speculated that local tissue injury in the setting of the malignancy may have allowed the organism to establish infection here. In a patient with AIDS and renal nocardiosis (Table 2, case 13), the authors suggested that local kidney damage caused by urolithiasis with subsequent hydonephrosis might have predisposed the patient to localized nocardiosis of the kidney [18].

As in our patient reported in Section 2, isolation of Nocardia species in the renal or adrenal region was the most common infection site amongst previous cases of abdominal or retroperitoneal nocardiosis (15 cases, 38\%). Further presentations include abscesses of the liver ( 7 cases, $18 \%$ ), spleen ( 3 cases, $8 \%$ ) and ovaries ( 2 cases, $5 \%$ ). Two thirds of the patients ( 26 cases, $66 \%$ ) had a least one immunocomprimising condition, in line with the literature on Nocardia species in general [1,7]. The mortality rate was high (36\%; 14 of 39 cases). Only 3 patients who died were reported to be immunocompetent. Unsuprisingly, mortality was higher in patients with disseminated nocardiosis than in those with isolated abdominal or retroperitoneal disease. Overall, abdominal/retroperitoneal nocardiosis seems to affect primarily adults, with only 4 cases $(10 \%)$ being diagnosed under the age of 18 years.

Accurate diagnosis of abdominal nocardiosis may be challenging due to the non-specific clinical and radiological findings. Additionally, Nocardia are often not visualized on initial stains, delaying clinical diagnosis and effective therapy [2]. Due to the slow growth of Nocardia species, adequate culture incubation of at least 5 days should be considered $[2,8]$. Abdominal nocardiosis is often misdiagnosed as tuberculosis [25,34] or abdominal malignancy such as adrenal [5], ovarian [19], thyroid [31] or renal carcinoma [2,39]. Misdiagnosis can lead to erroneous therapy including antituberculous treatment or even extensive surgery $[19,25]$. Suspected to have ovarian carcinoma, one published patient (Table 2, case 15) underwent total hysterectomy, salpingo-oophorectomy and local lymph node dissection before being diagnosed with abdominal nocardiosis [19].

Successful therapy typically included either percutaneous or surgical abscess drainage plus prolonged antimicrobial therapy ranging from 3 to 12 months. As for other forms of nocardiosis, effective antibiotics for abdominal Nocardia infections include trimethoprim-sulfamethoxazole (TMP-SMX), third-generation cephalosporins, extended-spectrum quinolones, carbapenemes, minocycline and linezolid [2]. In previous published cases of abdominal and retroperitoneal nocardiosis, TMP/SMX was the most commonly used antimicrobial agent (Tables 2 and 3). Given the typically prolonged antimicrobial therapy and the frequent use of combination therapy, kidney and liver function as well as potential pharmacological interactions should be closely monitored as demonstrated by our case report (Section 2). 
Table 2. Previously published cases of isolated abdominal/retroperitoneal nocardiosis.

\begin{tabular}{|c|c|c|c|c|c|c|c|c|c|}
\hline $\begin{array}{c}\text { Case } \\
n^{\circ} / \text { Reference }\end{array}$ & Year ** & Age & Sex & Species & Site & Immune Status & Therapy Regimen & $\begin{array}{l}\text { Therapy } \\
\text { Duration }\end{array}$ & Outcome \\
\hline $1[28]$ & 1976 & 49 & F & NR & Spleen abscess & Immunocompetent & Sulfisoxazole & NR & survived \\
\hline $2[9]$ & 1981 & 26 & $\mathrm{~F}$ & N. asteroides & Perirenal area * & Post renal transplant & Sulphatriad, fusidic acid & NR & survived \\
\hline $3[10]$ & 1983 & 11 & $\mathrm{~F}$ & N. asteroides & $\begin{array}{l}\text { Liver and kidney } \\
\text { abscesses }\end{array}$ & Immunocompetent & $\begin{array}{l}\text { Surgical resection, Amk, } \\
\text { TMP/SMX, Sulphadimidine }\end{array}$ & 12 weeks & survived \\
\hline $4[11]$ & 1986 & 76 & M & N. asteroides & Pancreas abscess & Immunocompetent & $\begin{array}{l}\text { Surgical resection, A/clav, Amk, } \\
\text { Orn, TMP/SMX }\end{array}$ & NR & survived \\
\hline $5[41]$ & 1990 & 19 & M & N. brasiliensis & $\begin{array}{l}\text { Cholecystitis and } \\
\text { Peritonitis }\end{array}$ & AIDS & NR & 7 days & died \\
\hline $6[12]$ & 1991 & 38 & M & N. asteroides & Left suprarenal abscess & AIDS & Surgical resection & NR & died \\
\hline 7 [13] & 1996 & 67 & M & N. asteroides & $\begin{array}{l}\text { Abdominal aortic } \\
\text { aneurysm }\end{array}$ & Immunocompetent & TMP/SMX, Amk & NR & survived \\
\hline $8[14]$ & 2003 & 42 & M & N. farcinica & Psoas abscess & Immunocompetent & TMP/SMX & 11 months & survived \\
\hline 9 [15] & 2004 & 37 & $\mathrm{~F}$ & NR & Abdominal abscess & M. Crohn & NR & NR & NR \\
\hline $10[5]$ & 2004 & 34 & M & N. asteroides & Left Adrenal abscess & AIDS & $\begin{array}{l}\text { Surgical resection, TMP/SMX, } \\
\text { Cef }\end{array}$ & NR & survived \\
\hline $11[16]$ & 2007 & 25 & $\mathrm{~F}$ & NR & $\begin{array}{l}\text { Bilateral kidney } \\
\text { abscesses }\end{array}$ & $\begin{array}{l}\text { Corticosteroids and } \\
\text { Azathioprin for SLE }\end{array}$ & Methylprednisolone & NR & died \\
\hline 12 [17] & 2007 & 44 & $\mathrm{~F}$ & NR & Jejunum & Post liver transplant & Surgical resection, Imi, Van & NR & died \\
\hline $13[18]$ & 2009 & 55 & M & NR & Kidney abscess & AIDS & Surgical resection & NR & NR \\
\hline $14[42]$ & 2010 & 83 & $\mathrm{~F}$ & N. veterana & Bowel abscess & Colon cancer & $\begin{array}{l}\text { Surgical resection, Cef, Gen, Met, } \\
\text { TMP/SMX }\end{array}$ & NR & survived \\
\hline 15 [19] & 2011 & 32 & $\mathrm{~F}$ & NR & $\begin{array}{l}\text { Bilateral abscesses of } \\
\text { ovaries and fallopian } \\
\text { tubes, omentum }\end{array}$ & Immunocompetent & $\begin{array}{l}\text { Surgical resection, Mino, } \\
\text { TMP/SMX, Amk, Line, Cip }\end{array}$ & 6 Months & survived \\
\hline $16[20]$ & 2012 & 59 & $\mathrm{~F}$ & N. farcinica & $\begin{array}{l}\text { Right adrenal } \\
\text { compartment }\end{array}$ & $\begin{array}{l}\text { Chronic hepatitis C, } \\
\text { post treatment for } \\
\text { lymphoma }\end{array}$ & Amk, Cefu, TMP/SMX & 4 months & died \\
\hline
\end{tabular}


Table 2. Cont.

\begin{tabular}{|c|c|c|c|c|c|c|c|c|c|}
\hline $\begin{array}{c}\text { Case } \\
\mathrm{n}^{\circ} / \text { Reference }\end{array}$ & Year ** & Age & Sex & Species & Site & Immune Status & Therapy Regimen & $\begin{array}{l}\text { Therapy } \\
\text { Duration }\end{array}$ & Outcome \\
\hline $17[21]$ & 2012 & 54 & $\mathrm{~F}$ & NR & Colon & Immunocompetent & $\begin{array}{l}\text { Surgical resection, antibiotics not } \\
\text { specified }\end{array}$ & NR & survived \\
\hline 18 [22] & 2013 & 30 & $\mathrm{~F}$ & NR & Left adnexal collection * & Immunocompetent & TMP/SMX, Amk & 6 months & survived \\
\hline $19[24]$ & 2016 & 58 & $\mathrm{~F}$ & N. farcinica & Colon, blood & $\begin{array}{l}\text { TNFa-antagonist for M. } \\
\text { Crohn }\end{array}$ & Van, Met, Imi, Amk, TMP/SMX & 12 months & survived \\
\hline $20[23]$ & 2017 & 59 & $\mathrm{M}$ & N. farcinica & Liver abscess & Post liver transplant & TMP/SMX, Amk, Imi & NR & survived \\
\hline $21[2]$ & 2018 & 63 & $\mathrm{~F}$ & N. paucivorans & Renal abscess & Post lung transplant & $\begin{array}{l}\text { Surgical resection, Imi, } \\
\text { TMP/SMX }\end{array}$ & $\begin{array}{l}9-12 \\
\text { months }\end{array}$ & NR \\
\hline $22[25]$ & 2019 & 63 & $\mathrm{M}$ & N. farcinica & Liver (hepatitis) & Immunocompetent & TMP/SMX, Amk & 4 weeks & died \\
\hline $23[26]$ & 2019 & 11 & $\mathrm{~F}$ & N. farcinica & Abdominal abscesses & Post renal transplant & TMP/SMX, Mer, Imi, Cila, Line & NR & NR \\
\hline $\begin{array}{l}24 \text { [present } \\
\text { report] }\end{array}$ & & 81 & $\mathrm{M}$ & N. paucivorans & Right adrenal space & $\begin{array}{l}\text { B-Cell Lymphoma, } \\
\text { Hypogammaglobulinemia }\end{array}$ & $\begin{array}{l}\text { Surgical resection, A/clav, } \\
\text { TMP/SMX, Amk, Cef, Mino, Cip }\end{array}$ & 6 months & survived \\
\hline
\end{tabular}

Abbreviations: AIDS = acquired immunodeficiency syndrome; Amk = amikacin; A/clav = amoxicillin + clavulanic acid; Cef $=$ ceftriaxone; Cefu = cefuroxime; Cila $=$ cilastin; Cip = ciprofloxacin; Gen = gentamycin; Imi = imipenem; Line = linezolid; Mer = meropenem; Mino = minocycline; NR = not reported; Orn = ornidazole; SLE = systemic lupus erythematodes;

$\mathrm{TNFa}=$ tumor necrosis factor alpha; TMP/SMX = trimethoprim-sulfamethoxazole; Van = vancomycin; * not further specified; ** Year of publication

Table 3. Previously published cases of disseminated nocardiosis with abdominal/retroperitoneal infection.

\begin{tabular}{|c|c|c|c|c|c|c|c|c|c|}
\hline $\begin{array}{c}\text { Case } \\
\mathbf{n}^{\circ} / \text { Reference }\end{array}$ & Year ** & Age & Sex & Species & Site & Immune Status & Therapy Regimen & $\begin{array}{l}\text { Therapy } \\
\text { Duration }\end{array}$ & Outcome \\
\hline $1[27]$ & 1975 & 13 & $\mathrm{M}$ & N. asteroides & $\begin{array}{l}\text { Lungs, liver, pancreas, } \\
\text { lymph nodes }\end{array}$ & Immunocompetent & $\begin{array}{l}\text { Surgical resection, } \\
\text { sulphadiazine, sulfisoxazole }\end{array}$ & 6 months & NR \\
\hline $2[29]$ & 1997 & 59 & $\mathrm{M}$ & N. otitidiscaviarum & $\begin{array}{l}\text { Thoracoabdominal } \\
\text { abscess, lung }\end{array}$ & HIV positive & $\begin{array}{l}\text { Surgical drainage, TMP/SMX, } \\
\text { Amk, Cefotaxime }\end{array}$ & NR & survived \\
\hline $3[30]$ & 1998 & 49 & $\mathrm{~F}$ & N. asteroides & $\begin{array}{l}\text { Left adrenal abscess, } \\
\text { spleen, lung }\end{array}$ & chronic steroids for RA & Surgical resection & NR & survived \\
\hline $4[34]$ & 2004 & 32 & $\mathrm{M}$ & N. brasiliensis & Lung, intestines & AIDS & Surgical resection & NR & died \\
\hline
\end{tabular}


Table 3. Cont.

\begin{tabular}{|c|c|c|c|c|c|c|c|c|c|}
\hline $\begin{array}{c}\text { Case } \\
\mathrm{n}^{\circ} / \text { Reference }\end{array}$ & Year ** & Age & Sex & Species & Site & Immune Status & Therapy Regimen & $\begin{array}{l}\text { Therapy } \\
\text { Duration }\end{array}$ & Outcome \\
\hline $5[17]$ & 2007 & 30 & $\mathrm{M}$ & N. asteroides & Lung, pleura, abdomen * & $\begin{array}{l}\text { Post kidney transplant, } \\
\text { chemotherapy, } \\
\text { corticosteroids }\end{array}$ & NR & NR & survived \\
\hline $6[31]$ & 2007 & 67 & $\mathrm{M}$ & N. asteroides & $\begin{array}{l}\text { Pancreas, omentum, } \\
\text { brain, lungs, thyroid }\end{array}$ & $\begin{array}{l}\text { Chronic steroids for } \\
\text { Still's disease }\end{array}$ & Mer, Amk, TMP/SMX & NR & died \\
\hline $7[32]$ & 2009 & 69 & $\mathrm{M}$ & N. farcinica & $\begin{array}{l}\text { Adrenal glands, brain, } \\
\text { lung, skin, muscle }\end{array}$ & Post liver transplant & TMP/SMX & 7 months & survived \\
\hline $8[6]$ & 2010 & 66 & $\mathrm{~F}$ & N. farcinica & $\begin{array}{l}\text { Brain, bilateral adrenal } \\
\text { abscesses, abdominal } \\
\text { lymph nodes }\end{array}$ & $\begin{array}{l}\text { aTNF-therapy for } \\
\text { Psoriasis }\end{array}$ & $\begin{array}{l}\text { Van, Amp, Mer, Voriconazole, } \\
\text { TMP/SMX, Line }\end{array}$ & 2.5 months & died \\
\hline $9[33]$ & 2010 & 61 & $\mathrm{~F}$ & N. nova & $\begin{array}{l}\text { Lung, skin, kidney, } \\
\text { pancreas, brain }\end{array}$ & $\begin{array}{l}\text { Chronic steroids and } \\
\text { Azathioprine for } \\
\text { ulcerative colitis }\end{array}$ & TMP/SMX & 1 year & survived \\
\hline 10 [35] & 2011 & 42 & $\mathrm{M}$ & N. concava & Lung, liver & $\begin{array}{l}\text { Chronic steroids for } \\
\text { polychondritis }\end{array}$ & $\begin{array}{l}\text { Sulphadiazine, Van, Imi, Cip, } \\
\text { Amk }\end{array}$ & 25 days & died \\
\hline $11[20]$ & 2012 & 68 & $\mathrm{~F}$ & N. farcinica & $\begin{array}{l}\text { Right kidney abscess, } \\
\text { brain abscess, lung }\end{array}$ & Anorexia nervosa & $\begin{array}{l}\text { Cef, Erythromycin, } \\
\text { TMP/SMX, Amk, Imi, Cip }\end{array}$ & 65 days & died \\
\hline 12 [36] & 2014 & 75 & M & N. farcinica & $\begin{array}{l}\text { Kidney, liver, spleen, } \\
\text { lung, brain }\end{array}$ & Immunocompetent & $\begin{array}{l}\text { Mer, Van, Cefepime, } \\
\text { Doxycycline, Acilovir }\end{array}$ & 3 days & died \\
\hline 13 [37] & 2015 & 59 & $\mathrm{~F}$ & N. cerradoensis & $\begin{array}{l}\text { Brain, skin, } \\
\text { retroperitoneum, lung }\end{array}$ & Post renal transplant & Mer, Amk & 3 months & survived \\
\hline $14[40]$ & 2016 & 37 & M & N. otitidiscaviarum & $\begin{array}{l}\text { Subcutaneous soft tissue, } \\
\text { liver, lung }\end{array}$ & Immunocompetent & Cef, TMP/SMX, Mino & NR & survived \\
\hline $15[38]$ & 2016 & 58 & M & N. nova & Lung, ileum & $\begin{array}{l}\text { B-cell Non-Hodgkin } \\
\text { lymphoma }\end{array}$ & TMP/SMX, Imi & 11 months & died \\
\hline $16[39]$ & 2018 & 12 & M & $\begin{array}{l}\text { N. elegans/aobensis/ } \\
\text { africana complex }\end{array}$ & Kidney, lung, brain & Immunocompetent & Pip/Taz, Amk, Imi, Cip & 6 days & died \\
\hline
\end{tabular}

Abbreviations: AIDS = acquired immunodeficiency syndrome; Amk = amikacin; Cef = ceftriaxone; Cip = ciprofloxacin; HIV = human immunodeficiency virus; Imi = imipenem; Line =

linezolid; Mer = meropenem; Mino = minocycline; NR = not reported; Pip/Taz = piperacillin-tazobactam; RA = rheumatoid arthritis; aTNF = anti-tumor necrosis factor therapy; TMP/SMX

$=$ trimethoprim-sulfamethoxazole; Van = vancomycin; ${ }^{*}$ not further specified; $* *$ Year of publication. 


\subsection{Peritoneal Dialysis-Related Nocardia Peritonitis}

Nocardia species is a rare cause of peritonitis in patients on continuous ambulatory peritoneal dialysis (CAPD), with 12 published cases in the literature, which we summarize in Table 4 [8,43-53]. Peritonitis is an important complication leading to technique failure, hospitalization and even mortality in CAPD patients [53]. Regular fluid exchange via the permanent percutaneous catheter exposes CAPD patients (but not patients on hemodialysis) to an increased risk for bacterial peritoneal contamination, which occasionally may include opportunistic pathogens such as Nocardia.

Identifying and treating Nocardia peritonitis may be difficult for several reasons. First, the clinical presentation of Nocardia peritonitis typically is no different from ordinary bacterial peritonitis [47]. Second, Nocardia may tend to form clumps in liquid environments, dispersing non-uniformly in peritoneal fluid, which may explain why Nocardia peritonitis can be difficult to identify [45]. Third, Nocardia grow slowly in culture, and the infection often presents as a "culture-negative" peritonitis, unresponsive to empirical antibiotic treatment. Extending the incubation time of the dialysate to a minimum of 10 days should be considered in cases of culture-negative and antibiotic-non-responsive peritonitis in CAPD-patients [8]. The finding of slow growing gram-positive rods in dialysate culture should raise the suspicion of Nocardia peritonitis [49]. Trimethoprim-sulfamethoxazole (TMP/SMX), often in combination with other antimicrobial agents, appears to be the treatment of choice $[50,51]$. Intraperitoneal antibiotic treatment is the preferred route of administration. In 5 of the 12 published CAPD patients with Nocardia peritonitis, intraperitoneal antibiotic administration was successful without removal of the catheter and therefore without technique failure. In the remaining cases, due to initial treatment failure, the PD-catheter was removed, and the patient switched to maintenance hemodialysis, and antibiotic treatment was given orally or intravenously until full resolution of the infection [48,51]. 
Table 4. Nocardia peritonitis in patients with continuous ambulatory peritoneal dialysis (CAPD).

\begin{tabular}{|c|c|c|c|c|c|c|c|c|c|}
\hline $\begin{array}{c}\text { Case } \\
\mathbf{n}^{\circ} / \text { Reference }\end{array}$ & Year ** & Age & Sex & Species & Cause of ESRD & Therapy Regimen & $\begin{array}{l}\text { Therapy } \\
\text { Duration }\end{array}$ & Outcome & $\begin{array}{l}\text { Catheter } \\
\text { Removal }\end{array}$ \\
\hline $1[43]$ & 1981 & 70 & M & N. asteroides & CIN & Ceph, Sulf & 6 weeks & survived & No \\
\hline $2[44]$ & 1990 & 60 & M & NR & PCKD & Ofl, Tob, Ceph, Van, Azt, TMP/SMX & 8 weeks & survived & No \\
\hline $3[45]$ & 1990 & 58 & $\mathrm{~F}$ & N. asteroides & NR & Ceft, Net, Van, TMP/SMX & NR & survived & Yes \\
\hline $4[46]$ & 1993 & 38 & M & N. asteroides & SLE & Ceph, TMP/SMX & 4 weeks & died & No \\
\hline $5[47]$ & 1994 & 80 & M & N. nova & NR & TMP/SMX & 3 weeks & survived & Yes \\
\hline $7[49]$ & 2003 & 68 & F & N. nova & Unknown & Cefa, Net, Ceft, Amk, Imi, TMP/SMX, Cef & 2 weeks & died & No \\
\hline $8[50]$ & 2005 & 35 & M & N. asteroides & CBUS & Van, Ceft, TMP/SMX, Amk, Cefu & 19 weeks & survived & No \\
\hline $9[51]$ & 2008 & 75 & M & NR & Type 2 DM & Van, TMP/SMX & 11 weeks & survived & Yes \\
\hline $10[8]$ & 2008 & 66 & M & N. asteroides & Type $2 \mathrm{DM}$ & Cefa, Gen TMP/SMX & 12 months & survived & Yes \\
\hline $11[52]$ & 2011 & 57 & M & N. asteroides & Type $2 \mathrm{DM}$ & Van, Ceft, TMP/SMX, Cef, Cip & 2 weeks & died & Yes \\
\hline $12[53]$ & 2016 & 13 & $\mathrm{~F}$ & N. asteroides & DGS & Van, Cip, Ceft, Line & 8 months & survived & Yes \\
\hline
\end{tabular}

Abbreviations: Azt = aztreonam; Cef = ceftriaxone; Cefa = cefazolin; Ceft = ceftazidime; Cefu = cefuroxime; Ceph = cephalotin; Cip = ciprofloxacin; CIN = chronic interstitial nephritis; CBUS = congenital bilateral ureteral stenosis; DGS= diffuse global sclerosis; DM = diabetes mellitus; ESRD = end-stage renal disease; Gen = gentamycin; Imi = imipenem; Line = linezolid; Net = netilmycin; NR = not reported; Ofl = ofloxacin; PCKD = polycystic kidney disease; SLE = systemic lupus erythematous; Sulf = sulfisoxazole; TMP/SMX = trimethoprim-sulfamethoxazole; Tob = tobramycin; Van = vancomycin; ${ }^{* *}$ Year of pulication. 


\section{Conclusions}

Human nocardiosis is a rare opportunistic disease affecting primarily immunosuppressed adults. However, one third of patients have no immunocompromising condition $[1,7,63]$. The acquisition route of abdominal nocardiosis typically is unclear. Hematologic dissemination after pulmonary or percutaneous inoculation and direct abdominal inoculation have been suggested to occur $[7,22,38]$. In addition, 12 cases of direct peritoneal inoculation in patients with continuous ambulatory peritoneal dialysis have been reported [8,43-53]. In abdominal nocardiosis, malignancy is often suspected due to the presence of an abdominal mass [2-6]. In our patient, an FDG-avid mass on FDG-PET led to suspicion of malignancy recurrence. Thus, biopsy of these masses and appropriate microbiological tests (including modified acid-fast staining, PCR, and culture) is crucial. Because of slow growth of Nocardia species, cultures should be incubated for at least 10 days to avoid false-negative results [8]. N. asteroides and $N$. farcinica are the most commonly isolated species in abdominal nocardiosis. N. paucivorans is a rare species leading to abdominal nocardiosis with only one previous case report, our patient thus representing the second published case. As in our patient, isolation of Nocardia species in the renal or adrenal region was the most common infection site amongst previous cases of abdominal or retroperitoneal nocardiosis. To date, there are no therapy guidelines for abdominal nocardiosis. In reported cases, successful therapy usually included either percutaneous or surgical abscess drainage plus prolonged combination antimicrobial therapy including TMP/SMX ranging from 3 to 12 months, as recommended for other forms of nocardiosis [69]. As demonstrated by our case report, liver and renal function as well as potential pharmacological interactions should be closely monitored during antimicrobial therapy.

Author Contributions: Conceptualization, L.T. and P.E.T.; histological report, K.D.M.; radiological report, R.H.; writing—original draft preparation, L.T. and P.E.T.; writing—critical review and editing, all authors. All authors have read and agreed to the published version of the manuscript.

Funding: No external funding was obtained for the preparation of the present review.

Conflicts of Interest: The authors declare no conflicts of interest.

\section{References}

1. Brown-Elliott, B.A.; Brown, J.M.; Conville, P.S.; Wallace, R.J., Jr. Clinical and laboratory features of the Nocardia spp. based on current molecular taxonomy. Clin. Microbiol. Rev. 2006, 19, 259-282. [CrossRef] [PubMed]

2. Biswas Roy, S.; Ross, M.D.; Patil, P.D.; Trepeta, R.; Bremner, R.M.; Panchabhai, T.S. Primary Nocardia Infection Causing a Fluorodeoxyglucose-Avid Right Renal Mass in a Redo Lung Transplant Recipient. Case Rep. Transplant. 2018, 2018, 9752860. [CrossRef] [PubMed]

3. Aliaga, L.; Fatoul, G.; Guirao, E.; Pena, A.; Rodriguez-Granger, J.; Cobo, F. Nocardia paucivorans brain abscess. Clinical and microbiological characteristics. IDCases 2018, 13, e00422. [CrossRef] [PubMed]

4. Shimizu, Y.; Tsuchiya, K.; Fujisawa, H. Nocardia paucivorans cerebellar abscess: Surgical and pharmacotherapy. Surg. Neurol. Int. 2019, 10, 22. [CrossRef]

5. Chong, Y.L.; Green, J.A.; Toh, K.L.; Tan, J.K. Laparoscopic drainage of nocardial adrenal abscess in an HIV positive patient. Int. J. Urol. 2004, 11, 547-549. [CrossRef]

6. Al-Tawfiq, J.A.; Al-Khatti, A.A. Disseminated systemic Nocardia farcinica infection complicating alefacept and infliximab therapy in a patient with severe psoriasis. Int. J. Infect. Dis. 2010, 14, e153-e157. [CrossRef]

7. Lerner, P.I. Nocardiosis. Clin. Infect. Dis. 1996, 22, 891-903; quiz 904-905. [CrossRef]

8. Kendrick-Jones, J.; Ratanjee, S.K.; Taylor, S.L.; Marshall, M.R. Nocardia asteroides peritoneal dialysis-related peritonitis: A case of successful treatment and return to peritoneal dialysis. Nephrol. Dial. Transplant. 2008, 23, 2693-2694. [CrossRef]

9. Lovett, I.S.; Houang, E.T.; Burge, S.; Turner-Warwick, M.; Thompson, F.D.; Harrison, A.R.; Joekes, A.M.; Parkinson, M.C. An outbreak of Nocardia asteroides infection in a renal transplant unit. Q. J. Med. 1981, 50, 123-135. 
10. Salfield, S.A.; Duerden, B.I.; Dickson, J.A.; Milner, R.D. Abdominal nocardiosis in a Sudanese girl. Eur. J. Pediatr. 1983, 140, 135-137. [CrossRef]

11. Meier, B.; Metzger, U.; Muller, F.; Siegenthaler, W.; Luthy, R. Successful treatment of a pancreatic Nocardia asteroides abscess with amikacin and surgical drainage. Antimicrob. Agents Chemother. 1986, 29, 150-151. [CrossRef]

12. Kim, J.; Minamoto, G.Y.; Grieco, M.H. Nocardial infection as a complication of AIDS: Report of six cases and review. Rev. Infect. Dis. 1991, 13, 624-629. [CrossRef]

13. Bocchetti, M.; Muzj, A.; Gargiulo, F.; Cipullo, P.; Amato, G. A case of abdominal aortic aneurysm infected by Nocardia asteroides. Biomed. Pharmacother. 1996, 50, 36. [CrossRef]

14. Smit, L.H.; Leemans, R.; Overbeek, B.P. Nocardia farcinica as the causative agent in a primary psoas abscess in a previously healthy cattle inspector. Clin. Microbiol. Infect. 2003, 9, 445-448. [CrossRef]

15. Saeed, S.; Varela, J.E.; Nelson, R.L.; Blend, M.J. An unusual case of intraabdominal nocardia abscess detected by Tc-99m HMPAO-labeled WBC study. Clin. Nucl. Med. 2004, 29, 270-271. [CrossRef]

16. Cassar, C.L. Nocardia sepsis in a multigravida with systemic lupus erythematosus and autoimmune hepatitis. Anaesth. Intensive Care 2007, 35, 601-604. [CrossRef] [PubMed]

17. Chedid, M.B.; Chedid, M.F.; Porto, N.S.; Severo, C.B.; Severo, L.C. Nocardial infections: Report of 22 cases. Rev. Inst. Med. Trop. Sao Paulo 2007, 49, 239-246. [CrossRef] [PubMed]

18. Pai, K.; Rao, L. Isolated renal nocardiosis in a patient with AIDS: Unusual presentation. Indian J. Urol. 2009, 25, 395-397. [CrossRef] [PubMed]

19. Patil, R.N.; Bafna, U.D.; Pallavi, V.R.; Rathod, P.S. Successful Intra-peritoneal Antibiotic Therapy for Primary Abdominal Nocardiosis in an Immunocompetent Young Female Masquerading as Carcinoma Ovary. Online J. Health Allied Sci. 2011, 10, 11.

20. De Montmollin, E.; Corcos, O.; Noussair, L.; Leflon-Guibout, V.; Belmatoug, N.; Joly, F.; Lefort, A. Retroperitoneal abscesses due to Nocardia farcinica: Report of two cases in patients with malnutrition. Infection 2012, 40, 93-96. [CrossRef] [PubMed]

21. Kamyab, A.; Fakhoury, J.D.; Sutkowski, R.; Drelichman, E.; Jacobs, M.J. Fulminant colitis secondary to nocardiosis. Int. J. Colorectal. Dis. 2012, 27, 841-842. [CrossRef] [PubMed]

22. Naha, K.; Dasari, S.; Vivek, G.; Prabhu, M. Primary abdominal nocardiosis masquerading as tubercular pelvic inflammatory disease in an immunocompetent individual. BMJ Case Rep. 2013, 2013. [CrossRef]

23. Hanchanale, P.; Jain, M.; Varghese, J.; V, J.; Rela, M. Nocardia liver abscess post liver transplantation-A rare presentation. Transpl. Infect. Dis. 2017, 19, e12670. [CrossRef]

24. David Turer, B.G.; Raghavendran, K. Fulminant Nocardia Colitis: A Case Report. Surgical Infect. Case Rep. 2016, 1, 69-71. [CrossRef]

25. Singh, S.; Verma, Y.; Pandey, P.; Singh, U.B. Granulomatous hepatitis by Nocardia species: An unusual case. Int. J. Infect. Dis. 2019, 81, 97-99. [CrossRef]

26. Acharya, R.; Amin, K.; Rajderkar, D.; Washam, M.; Pekkucuksen, N.; Mannemuddhu, S.; Upadhyay, K. Isolated abdominal nocardiosis in a pediatric renal transplant recipient. Pediatr. Transplant. 2019, 23, e13392. [CrossRef] [PubMed]

27. Idriss, Z.H.; Cunningham, R.J.; Wilfert, C.M. Nocardiosis in children: Report of three cases and review of the literature. Pediatrics 1975, 55, 479-484. [PubMed]

28. Chulay, J.D.; Lankerani, M.R. Splenic abscess. Report of 10 cases and review of the literature. Am. J. Med. 1976, 61, 513-522. [CrossRef]

29. Sandre, R.M.; Summerbell, R.C. Disseminated Nocardia otitidiscaviarum in a patient with AIDS. Can. J. Infect. Dis. 1997, 8, 347-350. [CrossRef]

30. Midiri, M.; Finazzo, M.; Bartolotta, T.V.; Maria, M.D. Nocardial adrenal abscess: CT and MR findings. Eur. Radiol. 1998, 8, 466-468. [CrossRef]

31. Indumathi, V.A.; Shivakumar, A.N.S. Disseminated nocardiosis in an elderly patient presenting with prolonged pyrexia: Diagnosis by thyroid abscess culture. Indian J. Med. Microbiol. 2007, 25, $294-296$. [CrossRef]

32. Jimenez-Galanes Marchan, S.; Meneu Díaz, J.C.; Caso Maestro, O.; Perez Saborido, B.; Moreno Elola-Olaso, A.; Abradelo Usera, M.; Fundora Suarez, Y.; Gimeno Calvo, A.; Moreno Molinero, V.; Garcia Reyne, A. Disseminated nocardiosis: A rare infectious complication following Non-heart-beating donor liver transplantation. Transplant. Proc. 2009, 41, 2495-2497. [CrossRef] 
33. Arora, G.; Friedman, M.; Macdermott, R.P. Disseminated Nocardia nova infection. South Med. J. 2010, 103, 1269-1271. [CrossRef] [PubMed]

34. John, M.A.; Madiba, T.E.; Mahabeer, P.; Naidoo, K.; Sturm, A.W. Disseminated nocardiosis masquerading as abdominal tuberculosis. S. Afr. J. Surg. 2004, 42, 17-19.

35. Hu, Y.; Zheng, D.; Takizawa, K.; Mikami, Y.; Dai, L.; Yazawa, K.; Fukushima, K.; Lu, C.; Xi, L. Systemic nocardiosis caused by Nocardia concava in China. Med. Mycol. 2011, 49, 662-666. [PubMed]

36. Xu, J.; Yachnis, A.T.; Malaty, I. An independent elderly woman with rapid onset of coma. JAMA Neurol. 2014, 71, 1043-1047. [CrossRef] [PubMed]

37. Piau, C.; Kerjouan, M.; Le Mouel, M.; Patrat-Delon, S.; Henaux, P.L.; Brun, V.; Morin, M.P.; Gautier, P.; Rodriguez-Nava, V.; Kayal, S. First case of disseminated infection with Nocardia cerradoensis in a human. J. Clin. Microbiol. 2015, 53, 1034-1037. [CrossRef]

38. Lim, M.Y.; Alker, A.P.; Califano, S.; Trembath, D.G.; Alby, K.; Gilligan, P.H.; Jamieson, K.; Serody, J.S.; Shea, T.C. Concurrent Disseminated Nocardiosis and GI Mucormycosis in a Stem-Cell Transplantation Recipient. J. Clin. Oncol. 2016, 34, e84-e86. [CrossRef]

39. Senard, O.; Blanot, S.; Jouvion, G.; Rodriguez-Nava, V.; Lortholary, O.; Join-Lambert, O.; Toubiana, J. Fulminant Nocardiosis Due to a Multidrug-Resistant Isolate in a 12-Year-Old Immunocompetent Child. Pediatrics 2018, 141, e20163131. [CrossRef]

40. Jiang, Y.; Huang, A.; Fang, Q. Disseminated nocardiosis caused by. Exp. Ther. Med. 2016, 12, 3339-3346. [CrossRef]

41. Bonacini, M.; Walden, J.M. Nocardia brasiliensis peritonitis in a patient with AIDS. Am. J. Gastroenterol. 1990, $85,1432-1433$.

42. Schlebusch, S.; Nimmo, G.; Carter, R. Bowel abscess with Nocardia veterana associated with colon carcinoma. Pathology 2010, 42, 306-307. [CrossRef] [PubMed]

43. Arfania, D.; Everett, E.D.; Nolph, K.D.; Rubin, J. Uncommon causes of peritonitis in patients undergoing peritoneal dialysis. Arch. Intern. Med. 1981, 141, 61-64. [CrossRef] [PubMed]

44. Chan, D.T.; Cheng, I.K.; Chan, P.C.; Mok, K.Y. Nocardia peritonitis complicating continuous ambulatory peritoneal dialysis. Perit. Dial. Int. 1990, 10, 99. [CrossRef] [PubMed]

45. Kaczmarski, E.B.; Wilkie, M.; Thornhill, C.; Oppenheim, B.A.; Ackrill, P. Problems encountered in diagnosis of Nocardia asteroides peritonitis complicating CAPD. Perit. Dial. Int. 1990, 10, 106. [CrossRef]

46. Lopes, J.O.; Alves, S.H.; Benevenga, J.P.; Salla, A.; Tatsch, I. Nocardia asteroides peritonitis during continuous ambulatory peritoneal dialysis. Rev. Inst. Med. Trop. Sao Paulo 1993, 35, 377-379. [CrossRef]

47. Recule, C.; Milongo, R.; Boiron, P.; Croize, J. Nocardia peritonitis complicating CAPD. Perit. Dial. Int. 1994, 14, 297-298.

48. Dwyer, K.M. Nocardia peritonitis and abdominal abscess complicating continuous ambulatory peritoneal dialysis. Nephrology 2001, 263-265. [CrossRef]

49. Chu, K.H.; Fung, K.S.; Tsang, W.K.; Chan, H.W.; Tong, K.L. Nocardia peritonitis: Satisfactory response to intraperitoneal trimethoprim-sulfamethoxazole. Perit. Dial. Int. 2003, 23, 197-198. [CrossRef]

50. Ortiz, A.M.; Rabagliati, R.; Machuca, E. Successful treatment of Nocardia asteroides peritonitis in a patient undergoing automated peritoneal dialysis and receiving immunosuppressive therapy. Adv. Perit. Dial. 2005, 21, 66-68.

51. Li, S.Y.; Yu, K.W.; Yang, W.C.; Chen, T.W.; Lin, C.C. Nocardia peritonitis-A case report and literature review. Perit. Dial. Int. 2008, 28, 544-547. [CrossRef] [PubMed]

52. Prasad, N.; Suresh, J.K.; Gupta, A.; Prasad, K.N.; Sharma, R.K. Nocardia asteroides peritonitis in peritoneal dialysis patients: Case report and review of the literature. Indian J. Nephrol. 2011, 21, 276-279. [CrossRef] [PubMed]

53. El-Naggari, M.; El Nour, I.; Al-Nabhani, D.; Al Muharrmi, Z.; Gaafar, H.; Abdelmogheth, A.A. Nocardia asteroides peritoneal dialysis-related peritonitis: First case in pediatrics, treated with protracted linezolid. J. Infect. Public Health 2016, 9, 192-197. [CrossRef] [PubMed]

54. Bosshard, P.P.; Abels, S.; Zbinden, R.; Böttger, E.C.; Altwegg, M. Ribosomal DNA sequencing for identification of aerobic gram-positive rods in the clinical laboratory (an 18-month evaluation). J. Clin. Microbiol. 2003, 41, 4134-4140. [CrossRef] 
55. Wayne, P. Performance Standards for Susceptibility Testing of Mycobacteria, Nocardia spp., and other Aerobic Actinomycetes, 1st ed.; CLSI supplement M62, ed.; Clinical and Laboratory Standards Institute: Wayne, NY, USA, 2018.

56. Hammoud, M.; Kraft, C.; Pulst-Korenberg, J.; Chenoweth, C.; Gregg, K.S. Disseminated Nocardia paucivorans infection in an immunocompetent host. Infection 2014, 42, 917-920. [CrossRef] [PubMed]

57. Minero, M.V.; Marin, M.; Cercenado, E.; Rabadan, P.M.; Bouza, E.; Munoz, P. Nocardiosis at the turn of the century. Medicine (Baltimore) 2009, 88, 250-261. [CrossRef]

58. Yassin, A.F.; Rainey, F.A.; Burghardt, J.; Brzezinka, H.; Mauch, M.; Schaal, K.P. Nocardia paucivorans sp. nov. Int. J. Syst. Evol. Microbiol. 2000, 50 Pt 2, 803-809. [CrossRef]

59. Wellinghausen, N.; Pietzcker, T.; Kern, W.V.; Essig, A.; Marre, R. Expanded spectrum of Nocardia species causing clinical nocardiosis detected by molecular methods. Int. J. Med. Microbiol. 2002, 292, 277-282. [CrossRef]

60. Eisenblatter, M.; Disko, U.; Stoltenburg-Didinger, G.; Scherubl, H.; Schaal, K.P.; Roth, A.; Ignatius, R.; Zeitz, M.; Hahn, H.; Wagner, J. Isolation of Nocardia paucivorans from the cerebrospinal fluid of a patient with relapse of cerebral nocardiosis. J. Clin. Microbiol. 2002, 40, 3532-3534. [CrossRef]

61. Wegerle, S.; Markus, A.; Weber, N.; Steffen, H.; King, B.; Eder, W.; Philipp, E.; Morresi-Hauff, A.; Haussinger, K.; Hoffmann, H. Pulmonary nocardiosis with trimethoprime/sulphamethoxazole-resistant Nocardia paucivorans in a patient with no signs of immunosuppression. Pneumologie 2007, 61, 46-51. [CrossRef]

62. Khan, S.H.; Sanche, S.E.; Robinson, C.A.; Pirouzmand, F. N. paucivorans infection presenting as a brain abscess. Can. J. Neurol. Sci. 2006, 33, 426-427. [CrossRef] [PubMed]

63. Gray, T.J.; Serisier, D.J.; Gilpin, C.M.; Coulter, C.; Bowler, S.J.; McCormack, J.G. Nocardia paucivorans-A cause of disseminated nocardiosis. J. Infect. 2007, 54, e95-e98. [CrossRef] [PubMed]

64. Monticelli, J.; Luzzati, R.; Maurel, C.; Rosin, C.; Valentinotti, R.; Farina, C. Brain Abscesses Caused by Nocardia paucivorans in a Multiple Myeloma Patient Treated with Lenalidomide and Dexamethasone: A Case Report and Review of Literature. Mediterr. J. Hematol. Infect. Dis. 2015, 7, e2015011. [CrossRef] [PubMed]

65. Delavari, N.; Than, K.D.; Chen, K.S.; McKeever, P.E.; Wang, A.C.; Pandey, A.S. Resolution of innumerable cerebral Nocardia paucivorans abscesses after medical management. J. Clin. Neurosci. 2016, 27, 175-177. [CrossRef] [PubMed]

66. Rafiei, N.; Peri, A.M.; Righi, E.; Harris, P.; Paterson, D.L. Central nervous system nocardiosis in Queensland: A report of 20 cases and review of the literature. Medicine (Baltimore) 2016, 95, e5255. [CrossRef]

67. Schiaroli, E.; Pasticci, M.B.; De Carolis, E.; Mello, E.; Pallotto, C.; Leli, C.; De Socio, G.V.; Baldelli, F.; Sanguinetti, M.; Mencacci, A. Diagnosis of Nocardia paucivorans central nervous system infection by DNA sequencing from paraffin-embedded tissue. Infez. Med. 2016, 24, 147-152.

68. Samuel, D.M.G.; Matta, M.; Podgore, J.K. Nocardia Paucivorans Empyema in a Patient with Suspected Silicosis. Am. J. Respir. Crit. Care Med. 2018, 2018, 197.

69. Wilson, J.W. Nocardiosis: Updates and clinical overview. Mayo Clin. Proc. 2012, 87, 403-407. [CrossRef]

(C) 2020 by the authors. Licensee MDPI, Basel, Switzerland. This article is an open access article distributed under the terms and conditions of the Creative Commons Attribution (CC BY) license (http://creativecommons.org/licenses/by/4.0/). 